\title{
Nonlinear Distortion Analysis Via Linear-Centric Models
}

\author{
Peng Li and Lawrence T. Pileggi \\ Department of Electrical and Computer Engineering \\ Carnegie Mellon University \\ Pittsburgh, PA 15213, USA \\ \{pli, pileggi\}@ece.cmu.edu
}

\begin{abstract}
An efficient distortion analysis methodology is presented for analog and RF circuits that utilizes linear-centric circuit models to generate individual distortion contributions due to the various circuit nonlinearities. The per-nonlinearity distortion results are obtained via a straightforward post-simulation step that is simpler and more efficient than the Volterra series based approaches and do not require the high order device model derivatives. For this reason the order of analysis can be significantly higher than that for a Volterra series implementation while fully accounting for all nonlinearity effects. The proposed methodology is not restricted to weakly nonlinear circuits, but can also analyze per-nonlinearity distortion for active switching mixers and switch capacitor circuits when they are modeled as periodically time-varying weakly nonlinear systems. While Volterra series have also been attempted for this same class of circuits, the requirement of device models for all of the high order model derivatives makes such analysis somewhat impractical. The proposed methodology provides important design insights regarding the relationships between design parameters and circuit linearity, hence the overall system performance. Circuit examples are used to demonstrate the efficacy of the proposed approach, and interesting insights are observed for RF switching mixers in particular.
\end{abstract}

\section{INTRODUCTION}

Circuit linearity is one of the most important design specifications for RF systems, and it can greatly impact the dynamic range of the corresponding communication system. Linearity is commonly characterized by the third order intermodulation intercept point (IIP3) and $1 \mathrm{~dB}$ compression point. These specifications are of great importance, particularly for RF applications, as the interference level tends to intensify. However, the actual nonlinear behaviors of complex RF and analog components are often inadequately understood, since observed nonlinear distortions can be due to a combination of several nonlinearities in the circuit. For this reason it is often desirable to analyze the individual distortion contributions to identify and adjust the dominant contributors as required to meet the design spec. However, obtaining these per-nonlinearity distortion contributions requires special analyses since simple superpositions are no longer valid for a nonlinear system.

Volterra functional series has been applied to analyze weakly nonlinear circuits such as amplifiers in [1]-[6]. Extensions to the important class of strongly nonlinear circuits such as active switching mixers and periodically switching networks have been made using time-varying Volterra series. These extensions are based on the time-invariant Volterra methods but treat the circuits being analyzed as periodically time-varying weakly nonlinear (PTVWN) systems [7][8]. For notational convenience in this paper we refer to the first type of circuits as weakly nonlinear, and the second class as PTVWN.
For Volterra series, the so-called nonlinear current method is employed to recursively solve for nonlinear responses or transfer functions in an increasing order fashion. At each step of nonlinear current method, a nonlinear excitation is generated for each circuit nonlinearity. When these nonlinear excitations are applied to the linearized circuit, a higher order circuit response is generated. In [4]-[6], while analyzing weakly nonlinear circuits, important interpretations of the nonlinear current method were developed such that individual distortion contributions could be extracted. When combined with symbolic analysis, Volterra series can be used, in theory, to generate very useful high-level circuit models. In practice, however, this process is very complex and computationally expensive [4]-[6]. Deriving analytical expressions for PTVWN circuits using time-varying Volterra series can quickly become intractable and require significant approximations[7][8]. It is possible to numerically compute the small-signal nonlinear circuit response using Volterra series with less cost, which involves repeatedly solving the linearized circuit with different inputs. In SPICE3[16], time-invariant Volterra series were applied to analyze distortions for weakly nonlinear circuits up to order three. In Volterra series, however, each circuit nonlinear characteristics is expressed as a Taylor series of its constitutive relationship. Therefore, accurate high order model derivatives are very difficult to obtain and often require additional model developments [17]. Furthermore, this difficulty severely limits the order of analysis that can be accurately performed. Typically, only a third order Taylor series is used to model a nonlinear element in the existing work.

Another type of closely related method, sensitivity analyses, when combined with steady-state simulation methods such as harmonic balance [18], can compute output incremental sensitivities w.r.t. design or process parameters at the converged circuit solution. But these methods are valid over a very small range of variation, and are more naturally suited for gradient guided optimizations, rather than for gaining insights in a design process.

At the opposite extreme for circuit analysis, progress in steadystate simulation methods [11][12] have made the simulation of ana$\log / \mathrm{RF}$ circuits with multi-tone excitations much more efficient than traditional time-domain transient analysis while providing accuracy that is limited only by the detailed device models they employ. However, knowing only the distortion levels observed at an output in a standard simulation run is often insufficient for providing design insights regarding how the overall linearity can be improved.

In this paper, we propose an efficient per-nonlinearity distortion analysis methodology for both weakly nonlinear circuits and PTVWN circuits. The method is based on a linear-centric circuit model for accurately capturing nonlinearities, and carried out as part of the circuit simulation process or as a simple post-simulation processing step while circumventing the aforementioned device model problem. This linear-centric model is motivated by an nonlinear iterative method, successive chord, in which constant linearizations for nonlinear elements are used to construct the Jacobian matrix [9]. 
The accuracy of the presented method is identical to that of the circuit-level simulation, yet individual distortion contributions up to an order only limited by circuit simulations are extracted using simple device model evaluations and one direct linear circuit solution. More importantly, we further show that the extracted total contributions are equivalent to what are produced in [4] for the weakly nonlinear case via much more complex analyses. The extracted quantities reveal almost the same amount of information regarding per-nonlinear contributions as the analysis in [4].To analyze PTVWN circuits, we further generalize the definition of distortion contributions in [4] to circuits with large nonlinearities by treating them as PTVWN under Volterra series setting, then show that linear-centric models produce the equivalent total distortion contribution information according to this more general definition. Circuit examples are shown to demonstrate the efficacy of our approach.

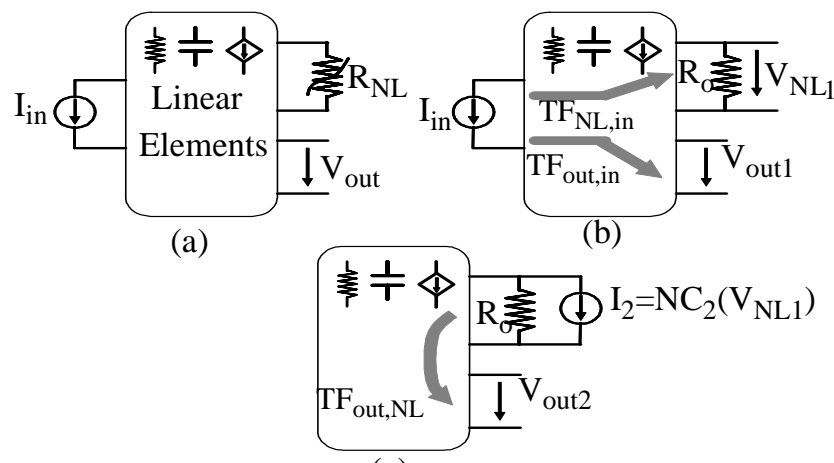

(c)

Fig. 1. (a) A nonlinear circuit, (b) first order response, and (c) second order response.

\section{VOLTERRA SERIES REVIEW}

In this section, we first review time-invariant Volterra series and its application to distortion analysis of weakly nonlinear circuits, such as weakly nonlinear amplifiers. In particular, the per-nonlinearity contribution analysis in [4] is discussed. Then, periodically time-varying Volterra series for periodically time-varying weakly nonlinear (PTVWN) circuits are reviewed. Ultimately, we extend the analysis in [4] to derive per-nonlinearity contribution analysis for PTVWN circuits under Volterra series setting.

\section{A. Time-Invariant Volterra Series}

Under certain conditions, the response of a weakly nonlinear circuit $y(t)$ can be expressed as a sum of responses at different or$\operatorname{ders}[1]-[3]$

$$
y(t)=\sum_{n=1}^{\infty} y_{n}(t)
$$

where, $y_{n}$ is the $\mathrm{n}$-th order response or distortion and can be related to circuit input by

$$
y_{n}(t)=\int_{-\infty}^{\infty} \ldots \int_{-\infty}^{\infty} h_{n}\left(\tau_{1}, \ldots, \tau_{n}\right) x\left(t-\tau_{1}\right) \ldots x\left(t-\tau_{n}\right) d \tau_{1} \ldots d \tau_{n} .
$$

In (2), $x(t)$ is the input to the system, and $h_{n}\left(\tau_{1}, \ldots, \tau_{n}\right)$ is the Volterra kernel of order $n$. The $\mathrm{n}$ dimensional Fourier transform of $\mathrm{n}$-th order kernel, $H_{n}\left(f_{1}, \ldots, f_{n}\right)$, is referred to as nonlinear transfer function of order $n$. Once the nonlinear transfer functions are available, various distortion quantities can be computed. For instance, it can be shown that the second and third order harmonic distortions are

$$
H D_{2}(f)=\frac{A^{2}}{4}\left|\frac{H_{2}(f, f)}{H_{1}(f)}\right|^{2}, H D_{3}(f)=\frac{A^{4}}{16}\left|\frac{H_{3}(f, f, f)}{H_{1}(f)}\right|^{2},
$$

respectively, where $f$ is the frequency of the sinusoidal input, and $A$ is the corresponding amplitude.

The nonlinear transfer functions or responses are computed using recursive nonlinear current method [1]-[3]. As an example, Fig. 1 demonstrates how the method is used to compute the second order response of the circuit shown in Fig. 1(a). For simplicity, Fig. 1(a) includes only a single nonlinear element, namely a voltage-controlled nonlinear resistor, $R_{\mathrm{NL}}$. First, a linearized circuit is computed at the dc operating point by replacing $R_{\mathrm{NL}}$ by its linearization $R_{\mathrm{O}}$. For the linearized circuit, we use $T F_{\text {out, in }}(\cdot), T F_{\mathrm{NL}, \text { in }}(\cdot)$, to denote the linear mappings relating the input to its voltage responses at the output and at the port of $R_{\mathrm{NL}}$, respectively. Also we use $T F_{\text {out, NL }}(\cdot)$ to denote the mapping from a current source applied at the port of $R_{\mathrm{NL}}$ to its voltage response at the output. The first order response is simply the response when the input is applied to the linearized circuit

$$
V_{N L 1}=T F_{N L, \text { in }}\left(I_{\text {in }}\right), V_{\text {out } 1}=T F_{\text {out, in }}\left(I_{\text {in }}\right) .
$$

When computing the $\mathrm{n}$-th order response or distortion $(\mathrm{n}>1)$, the external input is removed, and the nonlinear current source of order $n$, $I_{\mathrm{n}}$, is applied at the port of $R_{\mathrm{NL}}$ in the linearized circuit

$$
I_{n}=N C_{n}\left(V_{N L 1}, \ldots, V_{N L(n-1)}\right), V_{\text {outn }}=T F_{\text {out }, N L}\left(I_{n}\right) .
$$

In (5), $I_{n}$ is a function of lower order branch voltage responses, $V_{\mathrm{NLi}}$ $(\mathrm{i}<\mathrm{n})$, and the nonlinear characteristics of the nonlinearity. The latter one is expressed as a Taylor series of the nonlinear constitutive relationship expanded at the bias point. In general, there is one such nth order nonlinear current source for each nonlinearity, and the n-th order response is generated when all these nonlinear current sources superimpose on the linearized circuit.

A very important interpretation of (5) is that the contribution of a particular nonlinearity to the $\mathrm{n}$-th order distortion can be computed as the (linearized) circuit response when the corresponding n-th order nonlinear current source is applied [4]. Thus, this per-nonlinearity contribution information can be used to pinpoint the dominant distortion contributors in a design process.

As can be seen, to compute the total circuit response, the nonlinear responses of different orders are obtained by repeatedly solving the linearized circuit. In addition to the recursive nature of the above analysis, one will find that most device models are created to match the dc value and the first order small-signal behavior, but not the higher order derivatives. Therefore, additional model development is needed which is a significant impediment to using Volterra series for practical analog circuits. For a nonlinear amplifier under relatively large excitations, a high order Taylor series might be needed to model a nonlinear characteristics accurately. Under this case, a Volterra series analysis which captures only a first few lower order nonlinear effects cannot take into full account the nonlinear circuit behavior while generating higher order derivatives becomes increasingly difficult. 


\section{B. Periodically Time-Varying Volterra Series}

In PTVWN circuits such as switching mixers and switched-capacitor circuits, one large periodic excitation, LO signal or clock, causes strong nonlinear behavior. Input signals in the signal path are small and can be assumed to cause only weakly nonlinear distortions to the periodically varying operating point generated by LO or clock. This allows use of periodically time-varying Volterra series for distortion analysis. To reduce the amount of derivations, consider the time-domain Modified Nodal Analysis(MNA) formulation

$$
\left.f(y(t))+\frac{d}{d t} q(y(t))\right)+u(t)=0,
$$

where $y$ is the vector of node voltages and branch currents, $u$ is the vector of inputs, $f(\cdot)$ and $q(\cdot)$ are nonlinear functions relating currents of nonlinear resistors and nonlinear charges/fluxes with $y$, respectively. If a large periodic excitation $u_{L}(t)$ with a period of $T$ is applied to the system, then a periodic response $y_{L}(t)$ with the same period $T$ is produced. When a small signal input $u_{S}(t)$ is applied to the circuit along with $u_{L}(t)$, the (first order) small signal response due to $u_{S}(t)$ can be computed by linearizing the system along $y_{L}(t)$

$$
\frac{d}{d t}\left(C_{1}(t) y_{1}(t)\right)+G_{1}(t) y_{1}(t)+u_{S}(t)=0
$$

where $G_{1}(t)=\left.\frac{d f}{d y}\right|_{y=y_{L}(t)}, C_{1}(t)=\left.\frac{d q}{d y}\right|_{y=y_{L}(t)}$ are the $T$-periodic first order conductance and capacitance matrices. Note that (7) represents a linear periodically time-varying (LPTV) system. To consider weakly nonlinearities by the finite magnitude of $u_{S}(t)$, higher order terms are included in (7)

$$
\begin{gathered}
\frac{d}{d t}\left(C_{1}(t) y+C_{2}(t)(y \otimes y)+C_{3}(t)(y \otimes y \otimes y)+\ldots\right)+ \\
G_{1}(t) y+G_{2}(t)(y \otimes y)+G_{3}(t)(y \otimes y \otimes y)+\ldots+u_{S}(t)=0
\end{gathered}
$$

In (8) vector kronecker products of $y$ are used, and $G_{i}, C_{i}$ are the $i$ th order $T$-periodic conductance and capacitance matrices, respectively. Analogous to the time-invariant case, when the nonlinear current method is used to solve (8), responses at different orders are sought by recursively solving the LPTV circuit in (7) with different inputs. More specifically, the second and third order responses are determined by

$$
\begin{gathered}
\frac{d}{d t}\left(C_{1} y_{2}\right)+G_{1} y_{2}=-G_{2}(t)\left(y_{1} \otimes y_{1}\right)-\frac{d}{d t}\left(C_{2}\left(y_{1} \otimes y_{1}\right)\right) \\
\frac{d}{d t}\left(C_{1} y_{3}\right)+G_{1} y_{3}=-G_{3}\left(y_{1} \otimes y_{1} \otimes y_{1}\right)-G_{2}\left(y_{1} \otimes y_{2}+y_{2} \otimes y_{1}\right) \\
-\frac{d}{d t}\left(C_{3}\left(y_{1} \otimes y_{1} \otimes y_{1}\right)+C_{2}\left(y_{1} \otimes y_{2}+y_{2} \otimes y_{1}\right)\right)
\end{gathered}
$$

As can be seen, high order time-varying derivative matrices are required for the computation of nonlinear system response as a consequence of expanding nonlinear characteristics at every operating point. This requires an additional model development to provide accurate high order derivatives for all regions of operation. In [7], to use Volterra analysis, approximate device models suitable for analysis are extracted from BSIM3 models after certain effects are excluded.

Very similar to the time-invariant Volterra analysis, we interpret the nonlinear current method for extracting distortion contribution information in this time-varying Volterra series setting. The right hand sides of (9) and (10) can be recognized as the vector forms of nonlinear current sources of second and third orders, respectively. In general, each nonlinearity has an $i$ th order nonlinear current source which is a function of its time-varying nonlinear characteristics and lower order system responses. Just as in the time-invariant case, this nonlinear current component when applied to (7) generates a partial system response which can be interpreted as the nonlinearity's contribution to $i$ th order system response or distortion.

\section{DISTORTION ANALYSIS}

To avoid difficulties associated with direct application of Volterra series, the proposed method employs linear-centric circuit models for the nonlinear elements. This makes it possible to solve for each distortion contribution via straightforward device evaluations and one (time-varying) linear circuit solution. We first consider weakly nonlinear circuits, then generalize the method to PTVWN circuits.

\section{A. Weakly Nonlinear Circuits}

The linear-centric models employed here for distortion analysis are closely related to those from a linear-centric circuit simulation environment based on iterative successive chord (SC) nonlinear analysis [9][10]. For the SC method, constant linearizations for nonlinearities are used to construct a constant Jacobian matrix which is applied for all iterations. Nonlinear effects are only reflected in the right hand side vector via nonlinear device evaluations. Without loss of generality, each nonlinear element's contribution to the right hand side can be viewed as an added varying current source for each iteration. While the theoretical convergence rate for the SC method is only linear, it has been shown to outperform Newton-based methods due to the lower cost per iteration [10]. When the system of nonlinear equations satisfy convergence, the nonlinear circuit can be viewed as a constant linear circuit driven by external inputs plus right hand side contributions that represent the nonlinearities.

As an example, consider the voltage-controlled nonlinear resistor shown in Fig. 2 (a). In the vicinity of the bias point, its linearized resistance $R_{\mathrm{C}}$ represents the first order behavior. To model its behavior exactly, a time-varying current source $i_{\mathrm{C}}$ in parallel with $R_{\mathrm{C}}$ is

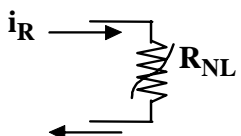

(a)

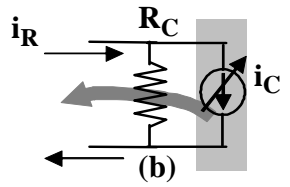

(b)
Fig. 2. (a) A nonlinear resistor, and (b) its linear-centric model

introduced, as depicted in Fig. 2 (b). The value of $i_{\mathrm{C}}$ is chosen in a way such that the circuit in Fig. 2 (b) is equivalent to that in Fig. 2 (a), i.e., the sum of the current which flows through $R_{\mathrm{C}}$ and $i_{\mathrm{C}}$ is always equal to the total branch current of $R_{\mathrm{NL}}$. We refer to the constant linearization of the nonlinear resistor, $R_{\mathrm{C}}$, as a chord, and the parallel time-varying current source, $i_{\mathrm{C}}$, as a chord current source. Here the name "chord" stems from the closely related SC method. In contrast to the traditional SC method, however, the linearization at the $d c$ bias point is always chosen for our distortion analysis in this paper. It is interesting to note that even though our linear-centric models are derived from a circuit simulation perspective, a very similar idea was suggested by Chua and $\mathrm{Ng}$ in [2].

Note that the actual value of $i_{\mathrm{C}}$ can be computed easily by sub- 
tracting the current which flows through $R_{\mathrm{C}}$ from total the branch current upon simulation convergence. It is straightforward to derive linear-centric models for other circuit elements such as transistors, etc.

The simple linear-centric model preserves important nonlinear behavior information in the chord current source, which when applied to the linearized circuit, generates the nonlinear distortion contribution due to the nonlinearity. In Fig. 3 we compare nonlinear current method and linear-centric modeling approach for the simple circuit example of Fig. 1. Although nonlinear responses are computed in an involved recursive fashion as explained in II.A., one key observation is that the total response is reached all at once by applying

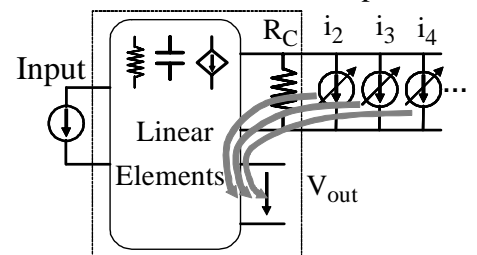

(a)

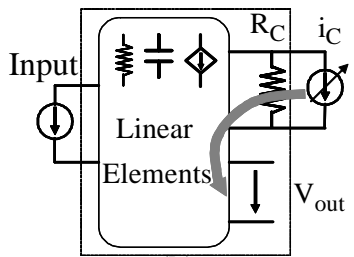

(b)
Fig. 3. (a) Nonlinear current method, and (b) linear-centric modeling

all nonlinear current sources of different orders and external inputs to the linearized circuit simultaneously. If we choose each chord of the linear-centric models according to the linearizations of nonlinearities at the bias point, it is not difficult to prove the following theorem using substitution theorem:

Theorem 1: If the following conditions are satisfied,

1.The weakly nonlinear condition for the applicability of Volterra series analysis is satisfied;

2.Truncation and numerical errors in the circuit simulation used to compute chord current sources are negligible; and,

3.Linearizations at dc bias are chosen as chords for all the nonlinearities.

Then under the same input excitations, the chord current source of any circuit nonlinearity is equal to the sum of nonlinear current sources at different orders of the same nonlinearity obtained from the time-invariant Volterra series analysis.

We omit the detailed proof of the Theorem 1 due to the page limits. However, Theorem 1 states that in our linear-centric approach, the chord current source represents the nonlinearity's total distortion contribution since it is the sum of nonlinear current sources of all orders. More specifically, to compute the contribution to any specific harmonic distortion term or intermodulation product, the corresponding frequency component of the chord current is multiplied by the linear transfer function from the port of a nonlinearity to the output.

It is worthy noting that when the distortion analysis is carried out in the frequency domain, as is almost always the case, merging contributions of different orders still preserves the frequency separation. Therefore, we can identify dominant distortion contributions on a per frequency basis. It is possible that a particular order of nonlinear contribution, say the third order, can locate at the same frequency as a higher order contribution, while our method provides the total distortion contribution for each nonlinearity at that frequen- cy. Furthermore, the Volterra series can compute the specific third order contribution due to its recursive nature. However, under the weakly nonlinear condition assumption, a higher order response is usually much smaller than a lower order response. Even when high order responses comprise a non-negligible contribution at a specific frequency, examining the total distortion is generally the objective for most applications. Additionally, our methodology provides results that are consistent with any circuit simulator that computes the total harmonic or intermodulation products at an output. Volterra series analysis can also distinguish contributions from nonlinear coefficients of different orders of a single nonlinearity, while our proposed linear-centric models do not provide such a distinction simply because they do not require device model derivatives beyond first order.

\section{B. PTVWN Circuits}

For periodically time-varying weakly nonlinear (PTVWN) circuits, assume that the only large-scale excitation (say LO in a mixer) to the circuit has a period of $T_{s}$, which gives rise to a periodically time-varying operating point for every circuit element. In this class of circuits, frequency translation is a wanted behavior, but the translation should be as linear as possible. If the nonlinear circuit is linearized along the varying operating point due to the large excitation, the resulting linear periodically time-varying(LPTV) circuit represents the "ideal" frequency translation. However, when a small input signal is applied, due to its finite amplitude, each nonlinear element encounters small perturbations along its ideal time-varying operating point that produce various distortion products.

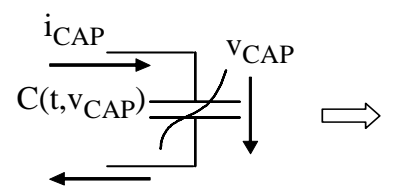

(a)
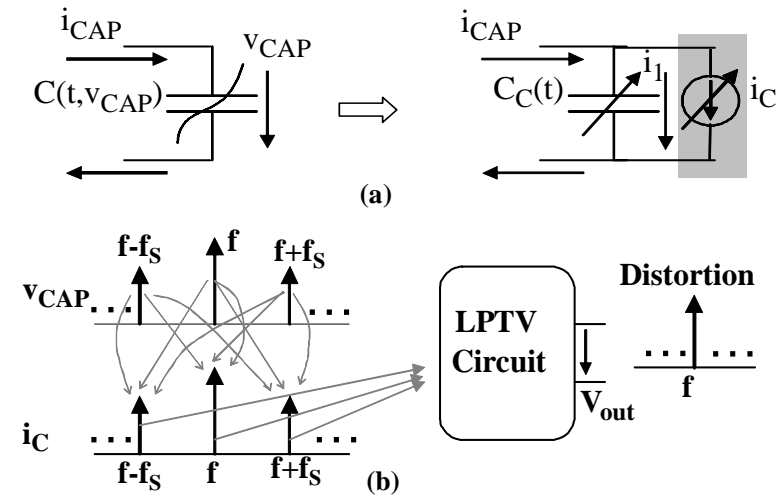

Fig. 4. (a) Linear-centric model of time-varying nonlinear capacitor, and (b) propagation of distortion contribution

To analyze the corresponding per-nonlinearity distortion contributions, we employ the linear-centric models shown in Fig. 4(a) for a time-varying nonlinear capacitor. This model is similar to that of the time-invariant case, except that a time-varying chord is used for the linearization of the nonlinear capacitor along its time-varying operating point. Using essentially the same arguments as those for theorem 1, we can show that the chord current source for any nonlinearity is equal to the sum of nonlinear current sources of different orders for the same nonlinearity obtained in time-varying Volterra series analysis. According to our interpretation of individual distortion contributions developed in II.B., as the chord current source propagates through the LPTV circuit, it generates the nonlinear distortions due to the nonlinearity.

\section{Computation of Distortion Contributions}

The simulation engine used in this paper is a successive chord it- 
erative method based harmonic balance simulator, SC-balance [10]. Note that even though the proposed distortion analysis can be most naturally incorporated with SC-balance, it is general enough to be used as a post-simulation processing step for any other standard steady-state simulators. In this section, we present how the per-nonlinearity distortion contributions can be computed under a harmonic balance environment.

Consider the nonlinear capacitor shown in Fig. 4(a), the total branch current upon convergence is

$$
I_{c a p}=\Omega \cdot \Gamma \cdot \operatorname{diag}(q(\cdot), \ldots, q(\cdot)) \cdot \Gamma^{-1} \cdot V_{c a p},
$$

where, $I_{c a p}=\left[I_{c a p}\left(\omega_{1}\right), \ldots, I_{c a p}\left(\omega_{N}\right)\right]^{T}$ is the Fourier coefficient vector of the current, $V_{\text {cap }}=\left[V_{\text {cap }}\left(\omega_{1}\right), \ldots, V_{\text {cap }}\left(\omega_{N}\right)\right]^{T}$ is the Fourier coefficient vector of the branch voltage, $q(\cdot)$ is the nonlinear function relating the charge to the voltage, $\Omega$ is the frequency domain differentiation operator, $\Gamma$ and $\Gamma^{-1}$ are DFT and inverse DFT matrices, and $N$ is the number of frequencies used in the simulation. Note (11) is used to evaluate a nonlinear capacitor at each harmonic balance iteration. Since the time-varying chord $C_{c}(t)$ is $T_{s}$-periodic, where $T_{s}=1 / f_{s}$ is the period of the large excitation to the circuit, it can be represented by a Fourier series expansion. The current flows though the chord can be expressed using phasors in quasi-periodic steady-state

$$
\begin{aligned}
i_{1}(t) & =\frac{d}{d t}\left(C_{c}(t) V_{c a p}(t)\right) \\
& =\sum_{l=1}^{N} \sum_{k=-\infty}^{\infty} j 2 \pi\left(k f_{s}+f_{l}\right) V_{c a p, l} C_{c, k} e^{j 2 \pi\left(k f_{s}+f_{l}\right) t}
\end{aligned}
$$

where $V_{c a p, l}$ is the phasor of $V_{c a p}(t)$ at frequency $f_{l}$ and $C_{c, k}{ }^{\prime} s$ are the Fourier coefficients of $C_{c}(t)$. The value of the chord current source $i_{c}(t)$ can be found by subtracting (12) from (11).

The per-nonlinearity distortion analysis can be carried out on a frequency by frequency basis. For example, in a two-tone test of a mixer, where the RF signal consists of two closely spaced sinusoidal tones $f_{1}$ and $f_{2}$. To compute the distortion contribution of the nonlinear capacitor to the output at $f=2 f_{1}-f_{2}-f_{s}$, where $f_{s}$ is the frequency of LO signal, one makes use of (11) and a truncated version of (12) to compute the frequency components of the chord current source at frequencies offset from $f$ by multiples of $f_{s}$. When these frequency components of the chord current propagate to the output through the LPTV network as in (7), they give rise to the distortion at the target frequency of $f$. Collectively, the distortion contribution can be expressed as

$$
\begin{aligned}
D(f, t) & =e^{j 2 \pi f t} \sum_{k=-K}^{K} T F\left(f, k f_{s}+f\right) \\
& \left(I_{c a p, k}-j 2 \pi\left(k f_{s}+f\right) \cdot \sum_{l=-K}^{K} V_{c a p, l} \cdot C_{c, k-l}\right)
\end{aligned}
$$

where, $C_{c, i}$ is the $i$ th Fourier coefficients of $C_{c}(t), V_{c a p, i}$ is the phasor of the branch voltage at frequency $f+i f_{s}, I_{c a p, i}$ is the phasor of branch current at frequency $f+i f_{s}$ given by (11), $T F\left(f, f+i f_{s}\right)$ is the linear periodic transfer function (LPTF) of the LPTV network which relates the current applied at the port of the capacitor at frequency $f+i f_{s}$ to its voltage response at the output node at frequency $f, K$ is the maximum frequency translations with respect to $f_{s}$ considered, respectively. Note that (11)-(13) should be modified accordingly for different types of nonlinearities.

LPTFs can be efficiently computed in the time domain by finite difference method [13] or in frequency domain by exploiting harmonic balance[14]. To extract the distortion contribution of every nonlinearity, LPTFs from every nonlinear port to the output need to be computed. LPTFs can be computed all at once by solving the adjoint network of the original LPTV network once. In this paper we use the method in [14] and the adjoint network formulation to efficiently compute LPTFs. The overall procedure is depicted in Fig. 4(b), which is very similar to the noise analysis when the noise folding effect is considered.

Compared to Volterra series, for our approach only the standard small-signal circuit parameters are required, and there is no need to directly evaluate high-order device derivatives -- which are difficult to obtain accurately. The analysis provides the same accuracy as the detailed circuit simulations. The dominant cost is reduced to a linear equation solution. Furthermore, our linear-centric analysis can proceed to high orders without difficulty in contrast to the practical third order limitation in Volterra series.

\section{RESULTS}

\section{A. A CMOS Two-stage Opamp}

A two-stage folded cascode opamp is shown in Fig. 5(a). For this example, the opamp is tested for harmonic distortion analysis in a typical closed loop configuration. A single tone sinusoidal input is applied from $1 \mathrm{KHz}$ to $100 \mathrm{MHz}$ and the opamp is simulated using the $S C$-balance simulator. Various distortion contributions are computed, including the dominant 3rd order harmonic distortion contributions which are shown in Fig. 5(b). The most dominant contributions are due to nonlinear drain currents of M19 and M20 for different frequency regions respectively. Since these two transistors form the second amplification stage where signal swings are greater than previous stage, the obtained result is conceptually intuitive. The analysis provides some quantitative measures of distortion contributions.

\section{B. A CMOS Differential Low Noise Amplifier}

A CMOS differential low noise amplifier (LNA) is shown in Fig. 6(a), where MOSFET pair M5 and M6 comprise the second gain stage. The LNA is tuned at $900 \mathrm{MHz}$ and a two tone intermodulation test is performed. Two input tones $f_{1}$ and $f_{2}$ are always $100 \mathrm{KHz}$ apart $\left(f_{2}=f_{1}+100 \mathrm{KHz}\right)$, and the LNA is simulated using $S C$-balance simulator and analyzed around a frequency neighborhood of $900 \mathrm{MHz}$.

Fig. 7 shows the most dominant contributions of the 3 rd order intermodulation product located at $2 f_{1}-f_{2}$. Since the differential pair 


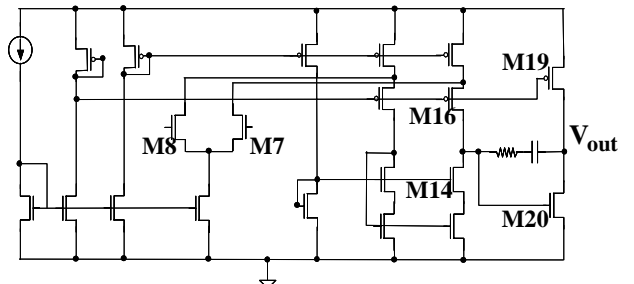

(a)

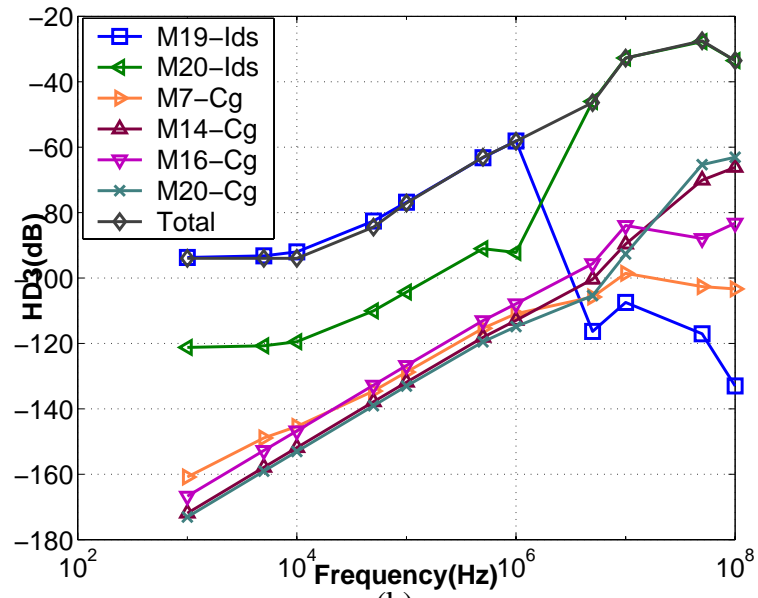

(b)

Fig. 5. (a) A CMOS opamp, and (b) dominate HD3 contributions

is completely symmetric, only the contributions of MOSFETs on one side are plotted. The most dominant IM3 contributions appear to be the nonlinear drain current and nonlinear gate capacitances of M5/M6. The gate capacitance contribution shown includes contributions due to nonlinear $C_{g s}, C_{g d}$, and $C_{g b}$. This result can be understood easily since large signal swings is expected to appear at the second stage of the amplifier. Further analysis shows that the even order distortion contributions from MOSFETs on the two sides of the different pair cancel out exactly. The observed suppression of even order nonlinear effects is what would be expected in a differential configuration when the exact symmetry between differential pair is maintained, as in this example.

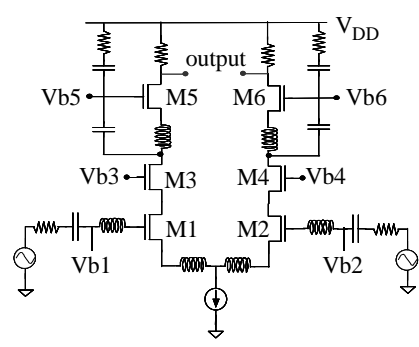

(a)

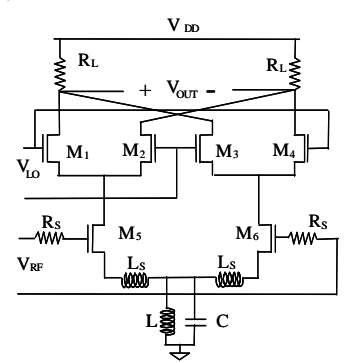

(b)
Fig. 6. (a) A CMOS low noise amplifier, and (b) a CMOS switching mixers

\section{A CMOS double-balanced mixer}

The proposed methodology for PTVWN circuits is applied to a double-balanced mixer in Fig. 6(b). Two-tone intermodulation tests are performed in which two tone frequency separation is $800 \mathrm{KHz}$ $\left(f_{1}=f_{2}+800 \mathrm{KHz}\right)$. The intermediate frequency is $70 \mathrm{MHz}$ with

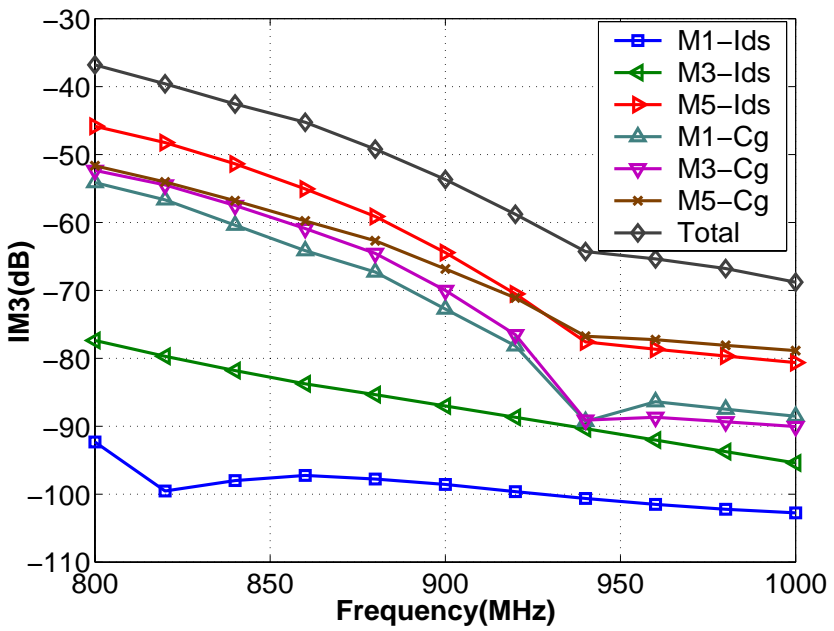

Fig. 7. Dominant IM 3 contributions of the LNA

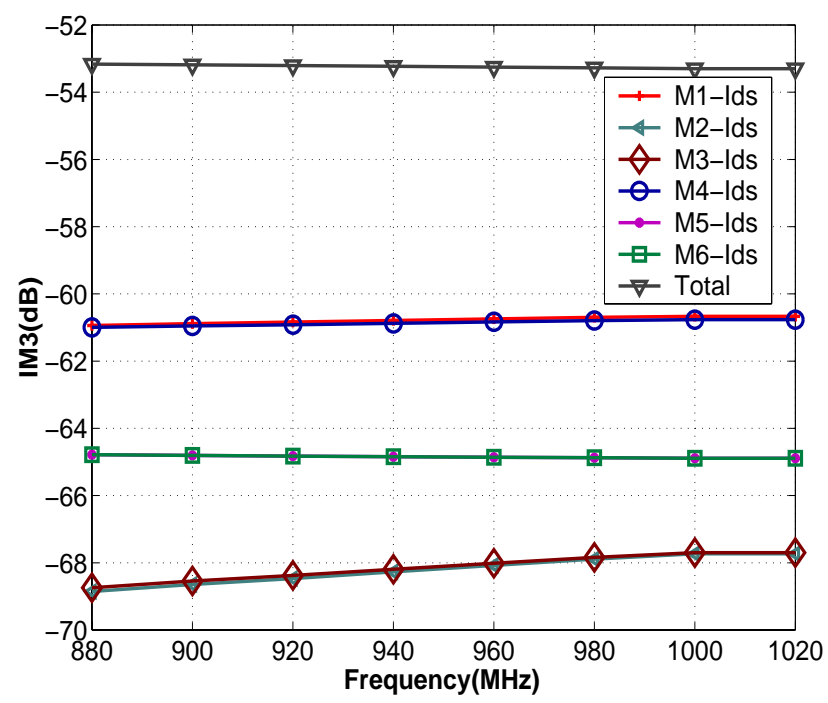

Fig. 8. Dominant IM3 Contributions of the Mixer

LO frequency $70 \mathrm{MHz}$ lower than $f_{1}$. The mixer is simulated using SC-balance as $f_{1}$ varies from $880 \mathrm{MHz}$ to $1.02 \mathrm{GHz}$, and the third order intermodulation distortion at $2 f_{1}-f_{2}-f_{L O}$ is analyzed at the output. The dominant distortion contributions of various circuit nonlinearities are plotted in Fig. 8.

In this example, the dominant third order intermodulation contributions are from the nonlinear drain currents of MOSFETs. It can be clearly seen that two MOSFET switches M1 and M4 contribute more distortion than MOSFETs M5 and M6 which form the transconductance stage. While typical design efforts often focus on the linearity of the transconductance stage assuming that M1-M4 are good switches [15] (primarily due to the difficulty of analyzing them via hand analysis), this detailed distortion analysis indicates that switching pairs can be the dominant distortion contributors. Similar results are reported in [7].

Another interesting observation is that contributions due to M2 
and M3 are less than those due to M1 and M4, despite the topological symmetry among them. This phenomenon is a consequence of the time-varying property of the circuit, since further analysis reveals that as the amplitude of the LO decreases toward zero, or as the circuit progressively approaches a nonlinear time-invariant system, the difference of distortion contributions among M1-M4 also decreases. At the time-invariant limit, the difference goes to zero. This result can be explained by realizing that the periodically timevarying operating conditions for these MOSFETs are out of phase with each other. This observation, however, cannot be exploited in the design since it is signal dependent, e.g., if the polarity of the LO is changed, then the distortion contribution of M1(M4) and that of M2(M3) are swapped.

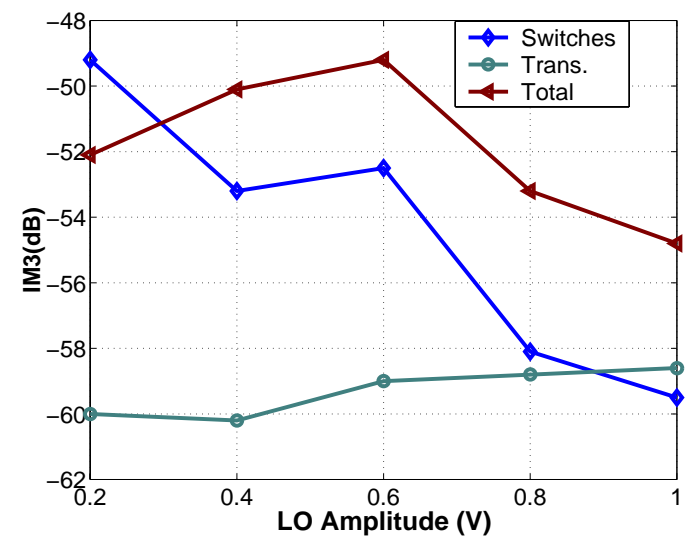

Fig. 9. Mixer IM3 Contributions as a function of LO amplitude

Next, we fix the frequencies of the two input tones at $900 \mathrm{MHz}$ and $900.8 \mathrm{MHz}$ respectively, and observe the third order intermodulation distortion at $71.6 \mathrm{MHz}$ as we vary the $\mathrm{LO}$ (peak to peak) amplitude. Fig. 9 plots the sum distortion contribution due to nonlinear drain currents of four MOSFET switches M1-M4, the sum distortion contribution due to nonlinear drain currents of transconductance stages M5-M6 and the total IM3, respectively. This plot demonstrates that for this particular circuit example, the distortion contribution of transconductance stages start to dominate only at sufficiently large LO drive. Therefore, distortion contributions of MOSFET switches must be properly considered, especially for small LO drive.

\section{CONCLUSIONS}

In this paper, an efficient per-nonlinearity distortion analysis methodology for both weakly nonlinear circuits and periodically time-varying weakly nonlinear circuits is presented. The adoption of linear-centric models for circuit nonlinearities makes it possible for us to perceive the weakly nonlinear circuit behavior as the linear "nominal" response plus the nonlinear "deviations" modeled by the chord current source for each nonlinearity. These chord current sources can be used to provide a systematic and quantitative analysis on the distortion contribution of each circuit nonlinearity in a manner critical design insight is provided. Most importantly, this insight is obtained at a very small added cost and in a fashion more efficient than the classical Volterra series analysis while not requiring any changes to the device models.

\section{ACKNOWLEDGEMENTS}

The authors would like to thank the Semiconductor Research Corporation (under contract 2000-TJ-779) for the financial support of this work.

\section{REFERENCES}

[1] J. Bussgang, L. Ehrman and J. Graham, "Analysis of nonlinear systems with multiple inputs," Proc. of IEEE, vol. 62, no. 8, pp. 1088-1119, August, 1974

[2] L. Chua and C. Ng, "Frequency domain analysis of nonlinear systems: general theory," IEE J. on Electronic Circuits and Systems, vol.3, no. 4, pp. 165-185, July 1979.

[3] D. Wiener and J. Spina, Sinusoidal analysis and modeling of weakly nonlinear circuits, Van Nostrand Reinhold, 1980.

[4] P. Wambacq and W. Sansen, Distortion analysis of analog integrated circuits, Kluwer Academic Publishers, 1998.

[5] P. Wambacq, G. Gielen, P. Kinget and W. Sansen, "High-frequency distortion analysis of analog integrated circuits," IEEE Trans. on Circuits and Systems-II: Analog and Digital Signal Processing, vol. 46. no. 3, March 1999.

[6] P. Wambacq, P. Dobrovolny, S. Donnay, M. Engels and I. Bolsens, "Compact modeling of nonlinear distortion in analog communication circuits," Proc. of DATE, 2000.

[7] M. Terrovitis and R. Meyer, "Intermodulation distortion in current-commutating CMOS mixers," IEEE J. of Solid-state Circuits, vol. 35, no. 10, pp. 1461-1473, October, 2000.

[8] F. Yuan and A. Opal, "Distortion analysis of periodically switched nonlinear circuits using time-varying volterra series," IEEE Trans. on Circuits and Systems-I: Fundamental Theory and Apps., vol. 48, no.6, pp. 726-737, June 2001.

[9] J. Ortega and W. Rheinboldt, "Iterative solution of nonlinear equations in several variables," Academic Press, 1970.

[10] P. Li and L. Pileggi, "A linear-centric modeling approach to harmonic balance analysis," Proc. of DATE, 2002.

[11] R. Telichevesky, K. Kundert, I. Elfadel and J. White, "Fast simulation algorithms for RF circuits," Proc. of IEEE Custom Integrated Circuits Conf., pp.437-444, 1996.

[12] P. Feldmann, B. Melville and D. Long, "Efficient frequency domain analysis of large nonlinear analog circuits," Proc. of CICC, 1996.

[13] R. Telichevesky, K. Kundert and J. White, "Efficient AC and noise analysis of two-tone RF circuits," Proc. of $33^{\text {rd }}$ DAC, 1996.

[14] J. Roychowdhury; D.Long and P. Feldmann, "Cyclostationary noise analysis of large RF circuits with multi-tone excitations," IEEE J. of Solid-State Circuits, vol. 33 issue 3, pp. 324 -336, March 1998.

[15] T. Lee, The design of CMOS radio-frequency integrated circuits, Cambridge University Press, 1998.

[16] J. Roychowdhury, "SPICE3 distortion analysis," Memo. no. UCB/ERL M89/48, Univ. of California, Berkeley, April, 1989.

[17] S. Maas and D. Neilson, "Modeling MESFETs for intermodulation analysis of mixers and amplifiers," IEEE Trans. on Microwave Theory and Techniques, volume 38, issue 12, pp. 19641971, December 1990.

[18] J. Bandler, Q. Zhang and R. Biernacki, "A unified theory for frequency-domain simulation and sensitivity analysis of linear and nonlinear circuits," IEEE Trans. on Microwave Theory and Techniques, vol. 36, no. 12, pp. 1661-1669, December 1988. 